\title{
GLOBAL ERROR CONTROL AND CPU-TIME MINIMIZATION IN DETERMINISTIC WAVE MODELS EXEMPLIFIED BY A FAST CONVOLUTION-TYPE MODEL
}

\begin{abstract}
Hemming A. Schäffer ${ }^{1}$
The convolution-type approach to deterministic wave modeling is briefly reviewed including both continuous and discrete formulations for the case of linear waves in one horizontal dimension (1DH). The associated discrete dispersion relation is presented and shown to accurately predict results of numerical simulations. This provides a tool for global error control and it is suggested that a similar approach is adopted for other deterministic wave models preferably along with a procedure for minimizing computational times while adhering to specified error tolerances. The discretization scheme for the 1DH convolution uses direct impulse-response-function sampling on a staggered grid. The explanation for the high accuracy of this approach is established and it is shown that the advantage does not carry over to the case of $2 \mathrm{DH}$. This calls for an entirely different method and it is sketched how a weighted least squares technique in wavenumber space might provide a satisfactory alternative in $2 \mathrm{DH}$. For variable depth this approach involves a slight distortion of physical space in order to retain a wavenumber-space formulation that resembles that of constant depth.
\end{abstract}

Keywords: waves, wave transformation, convolution, non-uniform discrete Fourier transform, weighted least squares, discrete dispersion relation, error control

\section{INTRODUCTION}

\section{General remark}

In coastal and ocean engineering, deterministic wave models are typically applied using rules of thumb to choose the values of the numerical parameters. Classical rules e.g. for Boussinesq-type models include the range of water depths and incident-wave periods, but not the physical size of the domain as would be needed to control global errors. Although this pragmatism often leads to satisfactory results, it is rarely optimal and may either lead to excessive CPU times or unacceptably large and sometimes unacknowledged errors.

This paper advocates for a paradigm shift in numerical-wave-modeling parameter selection from rules of thumb to global error control, preferably along with a minimization of CPU-time. The general ideas may be used in a variety of models, e.g. of the Boussinesq type, but the concept is sketched here for a so-called convolution-type deterministic wave model.

\section{The convolution model}

The convolution model relies on the time-stepping of the free-surface boundary conditions, while using an explicit convolution-type formulation in space to provide the required kinematics closure. This convolution is the central part of the model and it allows for full dispersion. In its complete form (still under development) the model allows for variable bathymetry, complex-shaped domains, and nonlinearity of arbitrary order.

In the continuous formulation, the convolution, which provides the vertical velocity at still water level, $w_{0}$, is an integral involving the horizontal velocity there and a depth-dependent continuous impulse response function. The impulse response function decays exponentially and in practice it vanishes at a horizontal distance of several water depths. In the discrete formulation, $w_{0}$ is simply determined as a weighted average of the discrete values of the horizontal velocity in the neighborhood. Thus, applying the discrete convolution operator is much like applying a finite difference stencil, although the influence area is depth-dependent and typically larger. The main challenge of the entire modeling approach is to express the convolution under general circumstances, first in a continuous and then in a discrete framework. As mentioned above, much of this work has been accomplished, but for the purpose of this paper, the focus is on the discrete formulations away from complicated boundaries.

Details for linear waves over a mildly sloping bottom (including discrete expressions) can be found in Schäffer 2009 for one horizontal dimension (1DH). Preliminary mild-slope developments for 2DH were given in Schäffer 2006 with the final formulation still to be published. Schäffer (2008a) looked into modifications of the convolution operator near odd boundaries using an analogy to the boundary integral equation method. The linear convolution operator reappears with successive use in the perturbation procedure adopted for nonlinear waves (Schäffer, 2005, 2008b). In the following sections

\footnotetext{
${ }^{1}$ SchäfferWaves, Sortedam Dossering 59 D st, DK-2100 Copenhagen $\varnothing$, Denmark
} 
the model is summarized in its linear form without accounting for the influence of complicated boundaries.

While the bulk of the theoretical developments have been completed in a continuous framework, detailed discrete expressions are only in place for 1DH. It turns out that the discretization approach used in $1 \mathrm{DH}$ is not viable in $2 \mathrm{DH}$. This paper takes a closer look at why and devises a possible solution.

\section{THE LINEAR SURFACE BOUNDARY CONDITIONS}

Restricting this paper to linear waves, the kinematic and dynamic surface boundary conditions in 2DH read

$$
\begin{gathered}
\eta_{t}=w_{0} \\
\mathbf{u}_{0, t}=-g \nabla \eta
\end{gathered}
$$

where $w_{0}$ and $\mathbf{u}_{0}$ is the vertical particle velocity and horizontal particle velocity vector, respectively, both taken at the still water level, $\eta$ is the free surface elevation, $g$ is acceleration of gravity, subscript $t$ denotes differentiation with respect to time, and $\nabla$ is the horizontal gradient operator.

The surface conditions can be integrated in time if $w_{0}$ is provided as a function of the other dependent variables. In a linear framework $w_{0}$ only depends on $\mathbf{u}_{0}$ and the bathymetry, and it may be explicitly given in terms of a convolution integral.

\section{THE MODEL IN 1DH}

\section{The continuous convolution}

The explicit convolution integral providing the kinematic closure for the time-stepping of the free surface boundary conditions (1)\&(2) may be expressed as

$$
w_{0}(x)=\int_{-\infty}^{\infty} R\left(x, x^{\prime}\right) u_{0}\left(x^{\prime}\right) d x^{\prime}=R * u_{0}
$$

where $*$ denotes the convolution operator, and the impulse response function for constant depth, $h$, is

$$
R\left(x, x^{\prime}\right)=\frac{-1}{2 h \sinh \left(\frac{\pi}{2} \frac{x^{\prime}-x}{h}\right)}
$$

The impulse response function is the inverse Fourier transform of $i \tanh (k h)$, where $k h$ is wavenumber times water depth and $i$ is the imaginary unit signifying the 90 degree phase shift between the horizontal and vertical particle velocity. Note that (3)\&(4) is wavenumber free and the formulation is valid for fully dispersive, irregular waves.

The mild-slope generalization of (4) is

$$
R\left(x, x^{\prime}\right)=\frac{-1}{2 h(x) \sinh \left(\frac{\pi}{2} \int_{x}^{x^{\prime}} \frac{1}{h\left(x^{\prime \prime}\right)} d x^{\prime \prime}\right)}
$$

The essence of this expression was derived by Matsuno (1993) using conformal mapping (see also Radder, 1992 and Otta et al, 1996), while Schäffer $(2005,2009)$ arrived at the result through infinite series differential operators that allow for generalization to $2 \mathrm{DH}$.

\section{The discrete convolution}

The spatial discretization is made using an equidistant, staggered grid of spacing $\Delta x$. The wavenumber range for the discrete model is thus $0 \leq k h<k_{N} h$, where $k_{N}=\pi / \Delta x$ is the Nyquist wavenumber. As usual, the grid spacing first and foremost has to be small enough for the shortest waves to be sufficiently resolved. If the resolution is fine enough for $k_{N} h$ to be in deep water, where $\tanh k_{N} h$ is sufficiently close to its limiting value of unity, then the discrete impulse response function is approximated very well simply by sampling the continuous impulse response function. As a rule of 
thumb, this requires $k_{N} h \geq \pi$ and thus $\Delta x / h \leq 1$, but the exact criterion depends on the required accuracy of the entire system in combination with the target wavenumber range, which is typically limited by the spatial differentiation of the surface elevation in (2).

The method of sampling the continuous impulse response function is used while observing the associated restrictions on $k_{N} h$, see also the section on aliasing. Using a staggered grid, we define the discrete variables as

$$
h_{p} \equiv h(p \Delta x) ; \quad \eta_{p} \equiv \eta(p \Delta x) ; \quad w_{p} \equiv w_{0}(p \Delta x) ; \quad u_{p} \equiv u_{0}\left(\left(p+\frac{1}{2}\right) \Delta x\right)
$$

and the discrete version of the continuous convolution in (3) becomes

$$
w_{p}=\sum_{q=p-m_{p}}^{p-1+n_{p}} r_{p, q} u_{q}
$$

Here $r_{p, q}$ are time-invariant, spatially variable filter weights to be computed prior to the time-stepping as

$$
r_{p, q} \equiv-\frac{\Delta x}{2 h_{p}} \frac{1}{\sinh \left(\frac{\pi}{2} S_{p, q}\right)}
$$

where

$$
S_{p, q} \equiv \int_{p \Delta x}^{\left(q+\frac{1}{2}\right) \Delta x} \frac{1}{h\left(x^{\prime \prime}\right)} d x^{\prime \prime}
$$

from is evaluated using the repeated midpoint rule. The half-widths of the discrete impulse response function, $\left(n_{p}, m_{p}\right)$, are determined as the smallest positive integers for which $s_{p, p-1+n_{p}} \geq s_{0}$ and $s_{p, p-m_{p}} \leq-s_{0}$, respectively. For constant depth, where $s=x / h, s_{0}=x_{0} / h$ defines the half width of the integration range in units of water depth. For variable depth, $s_{0}$ plays a similar role, but connects to $x$ by $d s / d x=1 / h$. For constant depth, $r_{p, q}$ and $s_{p, q}=(q-p+1 / 2) \Delta x / h$ depend on $q-p$, rather than $p$ and $q$ individually and the convolution reduces to a standard space-invariant process.

A detailed account for the discrete formulation including the time integration scheme (RK4) and an arbitrary-order scheme for the spatial derivative in the dynamic free surface boundary condition, (2), is given in Schäffer (2009).

\section{The numerical model}

The specific implementation of the convolution-type model is called SWIFT taken from Surface Waves Integral Fast Transformation. This name applies to the current 1DH version and will be retained for the coming extension to 2DH. The overall principle of SWIFT is to achieve a set up where the user can focus on error tolerances rather than numerical parameters. Specified tolerances for numerical damping and dispersion error are then taken as constraints, while the numerical parameters including the resolution are adjusted to minimize the computational effort. Traditionally, dispersive errors in Boussinesq equations have been assessed quantitatively by determining the errors of the embedded celerity relative to the fully dispersive target. However, the analysis can be taken one step further by evaluating the maximum accumulative phase error over a given distance. This is the approach needed for direct control (through the choice of numerical parameters) of how well simulations match the exact result e.g. looking at a time series at given distance from a common source of wave generation. A similar approach could well be taken for other models.

The model consists of the convolution, the first spatial derivative (of the surface elevation in the dynamic free surface boundary condition), and the time integration. In the discrete version each of these provides a source of error. The combined error can be determined by deriving the discrete dispersion relation. 
The consequence of the discretization is now analyzed for the case of constant depth. Taking $u_{p}=\sin (-k(p+1 / 2) \Delta x)$ should ideally produce $w_{p}=\tanh (k h) \cos (-k p \Delta x)$ and thus taking $p=0$ in (7) gives an error factor

$$
\gamma_{c} \equiv \frac{1}{\tanh (k h)} \sum_{q=-m_{p}}^{-1+n_{p}} r_{0, q} \sin (-k(q+1 / 2) \Delta x)
$$

where

$$
n_{p}=m_{p}=\left\lceil\frac{1}{2}+\frac{s_{0}}{\Delta x / h}\right\rceil=\left\lceil\frac{1}{2}+\frac{x_{0}}{\Delta x}\right\rceil
$$

and the subscript ' $c$ ' refers to 'convolution'. Here \lceil\rceil denotes the ceiling i.e. the smallest integer greater than or equal to the enclosed.

The discrete dispersion relation

Fourier analysis of the numerical scheme results in the discrete dispersion relation

$$
\left(\frac{\omega}{\gamma_{t}}\right)^{2}=g\left(\gamma_{s} k\right)\left(\gamma_{c} \tanh k h\right)
$$

where $\gamma_{c}$ is given by (10) and $\gamma_{t}$ and $\gamma_{d}$ are error factors from the time stepping and the discrete derivative operator, respectively. With reference to Schäffer (2009), a standard 4-stage, $4^{\text {th }}$ order Runge Kutta (RK4) scheme gives

$$
\gamma_{t}=\frac{1}{i \omega_{1} \Delta t} \log \sum_{n=0}^{4} \frac{1}{n !}\left(i \omega_{1} \Delta t\right)^{n}=1-\frac{(\omega \Delta t)^{4}}{120}+\frac{i(\omega \Delta t)^{5}}{144}+O(\omega \Delta t)^{6}
$$

where

$$
\omega_{1}^{2}=g\left(\gamma_{s} k\right)\left(\gamma_{c} \tanh k h\right)
$$

and an arbitrary-order finite difference scheme for a staggered grid gives

$$
\gamma_{s}=\frac{\sum_{n=0}^{p} 2 a_{2 n+1}\left(\sinh \frac{i k \Delta x}{2}\right)^{2 n+1}}{i k \Delta x}=1-\frac{a_{2 p+3}}{(-4)^{p+1}}(k \Delta x)^{2 p+2}+O(k \Delta x)^{2 p+4}
$$

The expansions are only accurate for small values of $\omega \Delta t$ and $k \Delta x$, respectively, and they are only listed to show the leading error.

For the standing-wave case of a fixed wavenumber (12) may be solved to get an angular frequency with a small imaginary part providing the damping in time. For a progressive wave of fixed frequency the solution gives a wavenumber with a small imaginary part providing the damping in space. These predictions provide a useful reference for practical model runs and serve to check the computer code.

\section{Minimizing the computational effort for given accuracy}

What now remains is to determine the numerical parameters that minimize the computational effort given the error tolerance. This minimization can accelerate the computation by an order of magnitude. Even so, the minimum is rather flat and a rough optimization is adequate. The optimization depends on the accuracy sought for a given physical problem. This section briefly outlines the approach.

The numerical parameters at hand are the temporal and spatial resolution, the finite-difference order parameter and the impulse response function truncation, $\left(\omega \Delta t, k \Delta x, p, s_{0}\right)$. Here the angular frequency and wavenumber correspond to the wave component for which error limits are specified. This would typically be the shortest wave to be modeled since this is prone to the largest error.

Although a more elaborate optimization is possible, the approach taken here is as follows. First choose the time step sufficiently fine to limit damping as required. Then determine the associated phase error and use a similar tolerance for the additional phase error caused by the discrete spatial operators. 
This total phase error is typically quite small and thus acceptable for most purposes. Smaller phase errors could be obtained by repeating the procedure for a smaller time step. Now optimize the computational effort while adjusting $\left(k \Delta x, p, s_{0}\right)$ under this phase error constraint. The rationale is to first balance dispersive errors in the temporal and spatial scheme to avoid excessive efforts in either scheme with little gain in accuracy. Simultaneous optimization appears to add more to the complexity than would be justified by the benefits.

The optimization of the parameters in the spatial operators is now made using the method of Lagrange multipliers by defining an objective function approximating the computational effort versus $\left(k \Delta x, p, s_{0}\right)$ and imposing a phase error constraint. To avoid the complications of the discrete order parameter, $p$, the optimization is made with respect to $\left(k \Delta x, s_{0}\right)$ within a $p$-loop, subsequently selecting the $p$-value giving the smallest minimum of the objective function. This minimum for the computational effort is rather flat and although it is crucial to choose $p$ near the optimum, the exact choice is not important.

\section{Model setup and sample result}

Fully reflective conditions are taken at both the up-wave and the down-wave boundaries, while wave generation as well as absorption is handled in their vicinity. Reflective boundary conditions are imposed requiring $\eta$ and $u_{0}$ to be even and odd functions around the boundary. In practice this is handled by (negatively) mirror imaging both the finite difference stencil applied to the surface elevation and the impulse response function for the convolution operator applied to $u_{0}$.

Wave generation near the left boundary and wave absorption near the right boundary are modeled by the use of relaxation zones (Bingham and Agnon, 2005). A relaxation zone accommodates a ramp function that specifies a gradual transition towards the boundary over which, at each time step, an increasing percentage of the numerically determined dependent variables are replaced by a prescribed theoretical solution. In case the theoretical solution is taken as zero for both dependent variables, the relaxation zone reduces to the commonly used numerical sponge layer. Now that the neighborhood of both ends of the domain are equipped with either a relaxation zone or a sponge layer, the present choice of reflecting end conditions is probably not significant.

The error estimates are made by comparing the numerical dispersion relation and the exact linear dispersion relation. The numerical dispersion relation accounts for the spatial scheme as well as the time stepping procedure. For real frequencies it has solutions in terms of complex wave numbers that embed both phase and amplitude errors. Accumulating the errors over the maximum wave travel distance of the modeling domain then provides the global errors estimates. Upper limits of the global errors are determined by the shortest waves for the largest water depth. Refined estimates could in principle account for the overall features of a variable bathymetry. However, no attempt was made to generalize the analysis in this direction as the benefits would not seem to match the added complexity.

Figure 1 shows results for a broad banded spectrum for which the highest frequencies correspond to four times the traditional deep-water limit, while the lowest frequencies are shallow water waves. For this case the model runs are orders of magnitude faster than real time.

\section{Why direct IRF sampling works well in 1DH}

In the discrete convolution leading to the dispersion relation (12) the convolution weights were determined by direct sampling of the impulse response function (IRF) on a staggered grid. Although Schäffer (2009) made a detailed analysis of the resulting accuracy, no attempt was made to explain how the approach could work so well. The missing explanation is provided in the following as an introduction to the case of two horizontal dimensions, where it turns out that direct IRF sampling is not feasible.

As mentioned in the section on the continuous formulation in $1 \mathrm{DH}$, the impulse response function (4) is the inverse Fourier transform of $i \tanh (k h)$. Since $i \tanh (k h) \rightarrow \pm i$ for $k h \rightarrow \pm \infty$, this Fourierspace transfer function is not band limited. Let us first look at the consequence of sampling while using a non-staggered equidistant grid. With reference to sampling theory the unlimited bandwidth makes direct sampling of the associated continuous impulse response function result in aliasing errors no matter how fine the sampling is. In general, let the transfer function $\Phi$ be expressed in terms of $k \Delta x$, then the discrete Fourier transform of the sampled impulse response function is the $2 \pi$-periodic function given by $F=\Sigma \Phi(k \Delta x-p 2 \pi)$ while summing up over all integer values of $p$. The target 
matches the term $p=0$, while all other positive and negative values of $p$ represent aliasing. Although the conventional limit of this series does not exist in the present case, the generalized limit (as may be obtained by ramping down $F$ starting at $k \Delta x>>\pi$ ) makes sense. For any resolution, the resulting transfer function would vanish at the Nyquist wavenumber, $k_{N} \Delta x=\pi$, and thus the accuracy would be very limited.

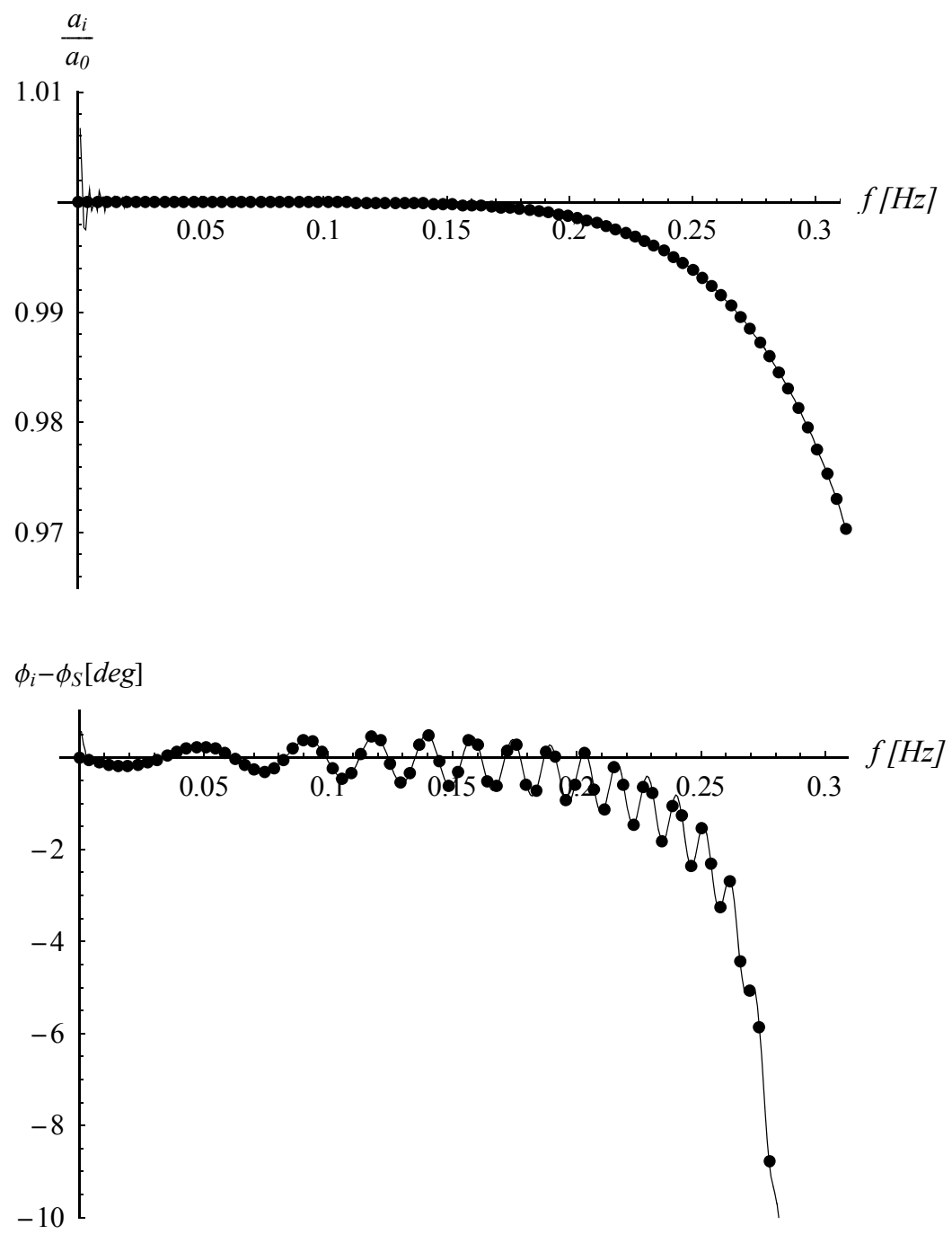

Figure 1. Decay of amplitude (top panel) and accumulated phase error (bottom panel) versus frequency for irregular waves that have travelled a distance of $2 \mathrm{~km}$ on a constant depth of $50 \mathrm{~m}$. The thin, full lines show results from the simulation. The dots show the prediction from the complex discrete dispersion relation. The results comply with specified accumulated error limits of $0.7 \%$ in amplitude and a few degrees in phase error both specified at $0.25 \mathrm{~Hz}$ frequency $(k h=12$., $h / L=2$.). The CPU time for the simulation was $13.3 \mathrm{~s}$ on a standard PC (and slightly faster for a variable depth simulation, not shown).

For a staggered grid, aliasing works differently. The effective grid size is now $\Delta x / 2$ and the discrete Fourier transform of the sampled impulse response function becomes the $4 \pi$-periodic function given by $F=\Sigma(-1)^{p} \Phi(k \Delta x-p 2 \pi)$. This is like the expression for the non-staggered grid but with an alternating sign which makes all the difference. Omitting the factor $i$ for simplicity, we look at $\Phi=\tanh (k h)=\tanh (k \Delta x h / \Delta x)$. Aliasing now vanishes if $k_{N} h$ is in deep water, which roughly requires $\Delta x / h \leq 1$. Figure 2 compares $\Phi$ with $F$ for $\Delta x / h=1$ and shows that within the Nyquist range $-\pi<k \Delta x<\pi$, where all wave energy must lie, there is no visible difference between the two. Figure 3 shows the same comparison, but for $\Delta x / h=2$. Significant aliasing now occurs, since 
$\tanh (k h)$ only comes close to its horizontal asymptotes somewhat outside the Nyquist range. For $\Delta x / h=3$ (see Figure 4), aliasing ruins the match for all wave numbers and the approach becomes inadequate.

In conclusion, the method of direct impulse response function sampling on a staggered grid is successful owing to that fact that $\tanh (k h)$ converges very fast (viz. exponentially) to its constant asymptotic values. Under these circumstances $\Delta x / h$ can be chosen to ensure any desired accuracy within the entire Nyquist wavenumber range. If $\Delta x / h$ is chosen too large or if the target transfer function had not shown fast convergence, then aliasing errors would ruin the accuracy, potentially for all wavenumbers.

For variable depth (mild slopes) the method of directly sampling the continuous impulse response function is very convenient, since while keeping $\Delta x$ constant, the sampling is just made with a slightly varying depth-scaled interval, cf. the change of argument for the Sinh-function from the constant-depth case of (4) to the mild-slope case of (5).

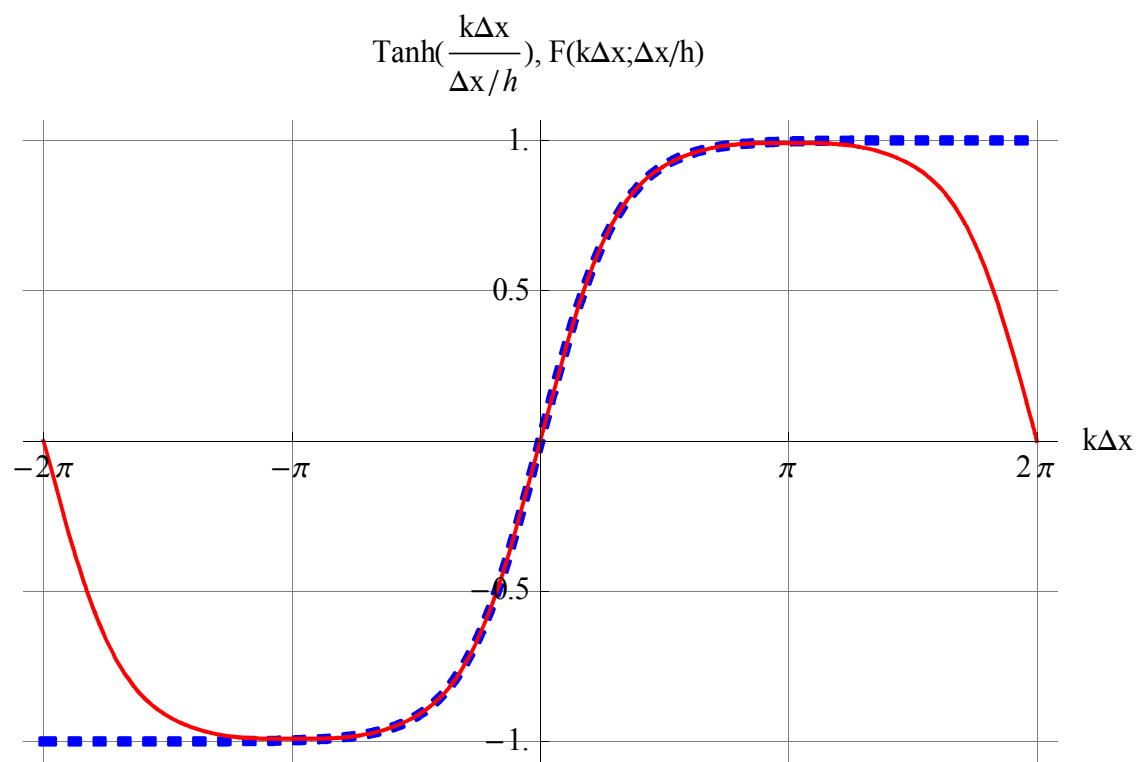

Figure 2. Imaginary part of target transfer function, $i \tanh (k h)$, (blue, dashed), along with the imaginary part of the transfer function corresponding to direct staggered sampling of the continuous impulse response function (red) for $\Delta x / h=1$. There is no visible aliasing within the Nyquist wavenumber range $-\pi<k \Delta x<\pi$.

\section{THE CONVOLUTION IN 2DH}

\section{Why direct IRF sampling is not feasible in 2DH}

In $2 \mathrm{DH}$, one option is to seek a formulation that resembles the one used above in $1 \mathrm{DH}$. The convolution would then involve the horizontal velocity vector, $\mathbf{u}_{0}$, and the target transfer function would be the vector function $(\mathbf{k} / k) i \tanh (k h)$, where $k$ is the magnitude of the wavenumber vector, $\mathbf{k}=\left(k_{x}, k_{y}\right)$. Taking the $x$-component as example, the target transfer function would be $k_{x} / \sqrt{k_{x}^{2}+k_{y}^{2}} i \tanh \left(\sqrt{k_{x}^{2}+k_{y}^{2}} h\right)$ and staggering would apply to the $x$-direction (in which the impulse response function is odd) and not to the $y$-direction (in which the impulse response function is even). For any $k_{y}$, this function has the same asymptotic values for $k_{x} h \rightarrow \pm \infty$ as for the 1DH case. However, in general (i.e. for $k_{y} \neq 0$ ) the function converges very slowly to the asymptotic values. With reference to the conclusion of the aliasing discussion for the $1 \mathrm{DH}$ case, this makes significant aliasing errors inevitable. Another source of aliasing (still looking at the $x$-component of the impulse response function and transfer function) lies in the sampling in the $y$-direction. Practical examples (not shown) confirm that the approach is indeed unfeasible. 


$$
\operatorname{Tanh}\left(\frac{\mathrm{k} \Delta \mathrm{x}}{\Delta \mathrm{x} / h}\right), \mathrm{F}(\mathrm{k} \Delta \mathrm{x} ; \Delta \mathrm{x} / \mathrm{h})
$$

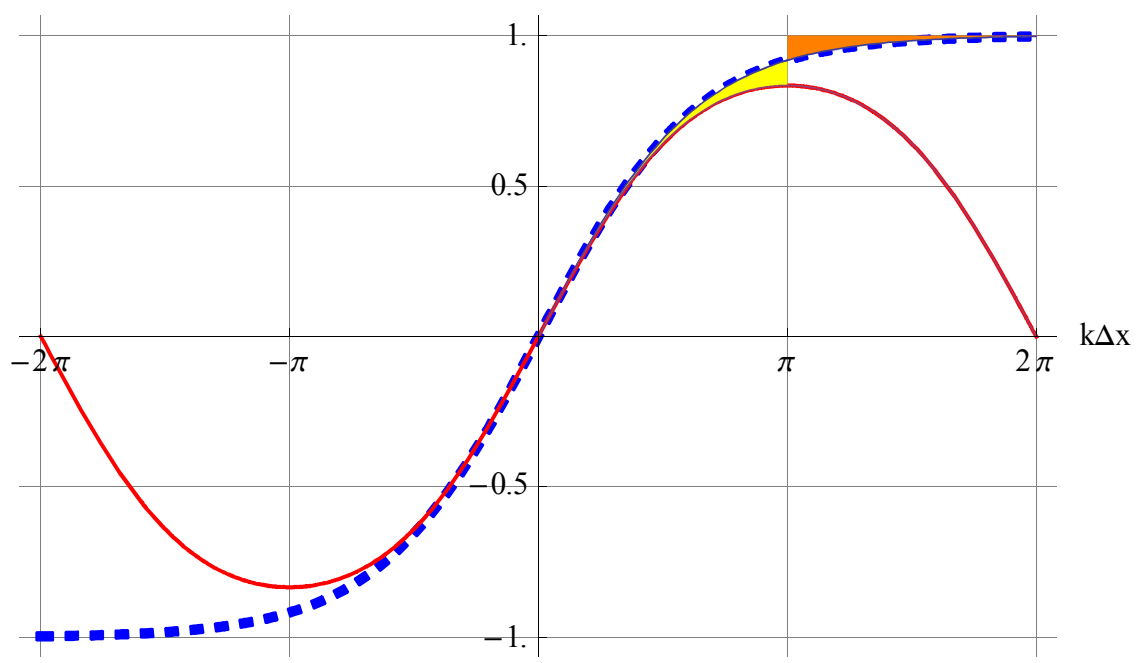

Figure 3. Like Figure 2, but for $\Delta x / h=2$, which gives pronounced aliasing as illustrated by the orange area folding to the yellow area.

$$
\operatorname{Tanh}\left(\frac{\mathrm{k} \Delta \mathrm{x}}{\Delta \mathrm{x} / h}\right), \mathrm{F}(\mathrm{k} \Delta \mathrm{x} ; \Delta \mathrm{x} / \mathrm{h})
$$

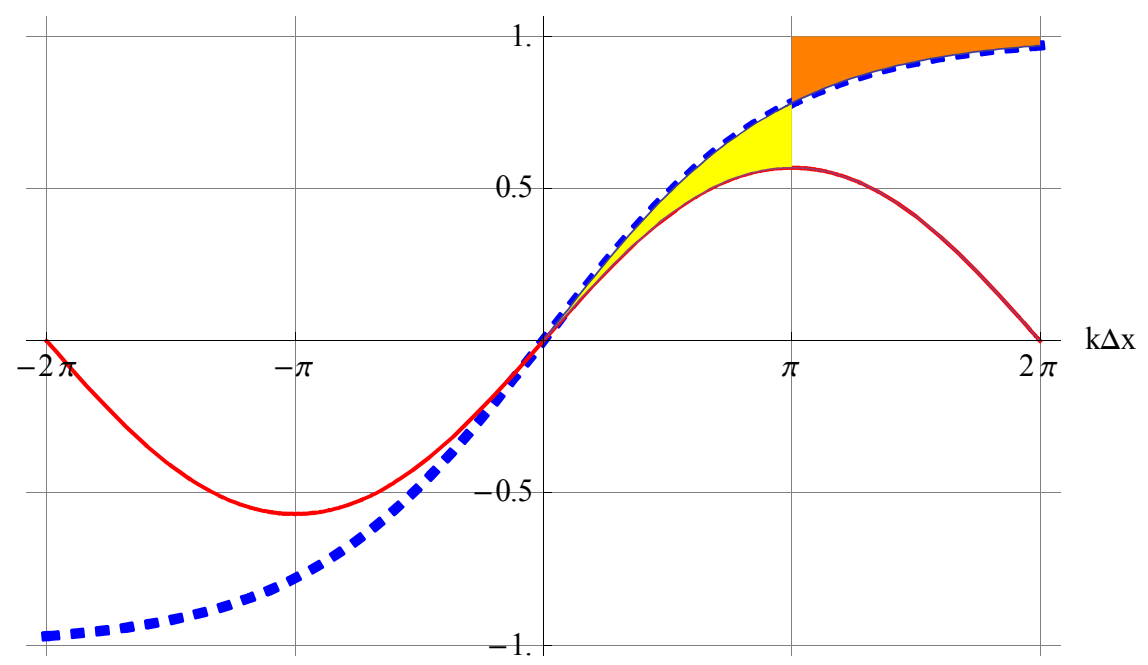

Figure 4. Like Figure 3, but for $\Delta x / h=3$, for which aliasing now destroys the accuracy for the entire range $-\pi<k \Delta x<\pi$.

\section{An alternative approach to the discrete convolution}

The unsuccessful attempt to generalize the highly accurate 1DH approach calls for an entirely different approach in 2DH. Abandoning the continuous impulse response function as the starting point for the discretization, the focus is now shifted to the target transfer function. The property of $\tanh (\mathrm{kh})$ of fast convergence to the asymptotic values as utilized in $1 \mathrm{DH}$, is no longer relevant and it turns out convenient to change to a scalar formulation, where the operand for the convolution operator is $\nabla \cdot\left(h \mathbf{u}_{0}\right)$ while the target transfer function is $\tanh (k h) /(k h)$. This makes one convolution suffice even in $2 \mathrm{DH}$ and the attained rotational symmetry in $\mathbf{k}$-space helps to simplify the following steps. For constant depth, the most apparent option is to determine the convolution coefficients as the 2D Fourier series coefficients of the doubly periodic function defined by $\tanh \left(\sqrt{k_{x}^{2}+k_{y}^{2}} h\right) /\left(\sqrt{k_{x}^{2}+k_{y}^{2}} h\right)$ over $-\pi<k_{x} \Delta x<\pi$ and $-\pi<k_{y} \Delta y<\pi$. Aliasing plays no role in the approach and the accuracy is flexible 
as it is only restricted by limiting the number of Fourier coefficients retained. The Fourier coefficients would make up the convolution stencil. This approach has the advantage over the one used in 1DH that the depth can be small compared to the physical-space resolution, i.e. the requirement that $\Delta x / h$ stays les than about unity no long applies. However, more important, this approach has the serious problem that in its primitive form, it is not applicable for variable depth. To solve this problem, we make the following assertions

1. The Fourier series approach corresponds to a least squares fit to the $\mathbf{k}$-space transfer function.

2. Variable depth can be handled using the same transfer function, but corresponding to a slightly distorted physical space, where the distortion resembles that of the $1 \mathrm{DH}$ case as detailed below.

This suggests a generalized approach, where the wavenumber-space transfer function corresponding to convolution coefficients on a slightly distorted grid makes a least squares fit to the target transfer function. Non-uniform weights may be applied in the least squares procedure, which amounts to solving a linear system of equations. The size of this system is modest, since it involves only the convolution weights to be applied at the given point. In the special case of uniform weights and constant depth, the system matrix reduces to the identity matrix and the convolution coefficients are merely the Fourier series coefficients as mentioned above.

With reference to the temporary formulation of the variable-depth case in 2DH by Schäffer (2006), the distorted coordinates, $(\tilde{x}, \tilde{y})$, are defined in continuous space through their polar counterparts $(s, \gamma)$, where $s=s(x, y)$ is given by

$$
|\nabla s|=\frac{1}{h}
$$

and $\gamma=\gamma(x, y)$ is orthogonal to $s(x, y)$,

$$
\nabla s \cdot \nabla \gamma=0
$$

Taking $(r, \theta)$ as local polar coordinates in the original physical space, we get to mild-slope accuracy

$$
s=\frac{r}{h}\left(1+\frac{1}{2} \frac{r}{h} \nabla h \cdot \nabla r\right)
$$

and

$$
\gamma=\theta+\frac{1}{2} \frac{r}{h} \nabla h \cdot r \nabla \theta
$$

An example is given in brief as follows. With $h_{0}$ denoting the water depth at the impulse, let the bathymetry be a plane slope according to

$$
\frac{h}{h_{0}}=1+\nabla h \cdot \frac{\mathbf{x}}{h_{0}} ; \quad \nabla h=\frac{1}{10}\left(\frac{2}{\sqrt{5}}, \frac{1}{\sqrt{5}}\right)
$$

and let

$$
\frac{\Delta x}{h_{0}}=1
$$




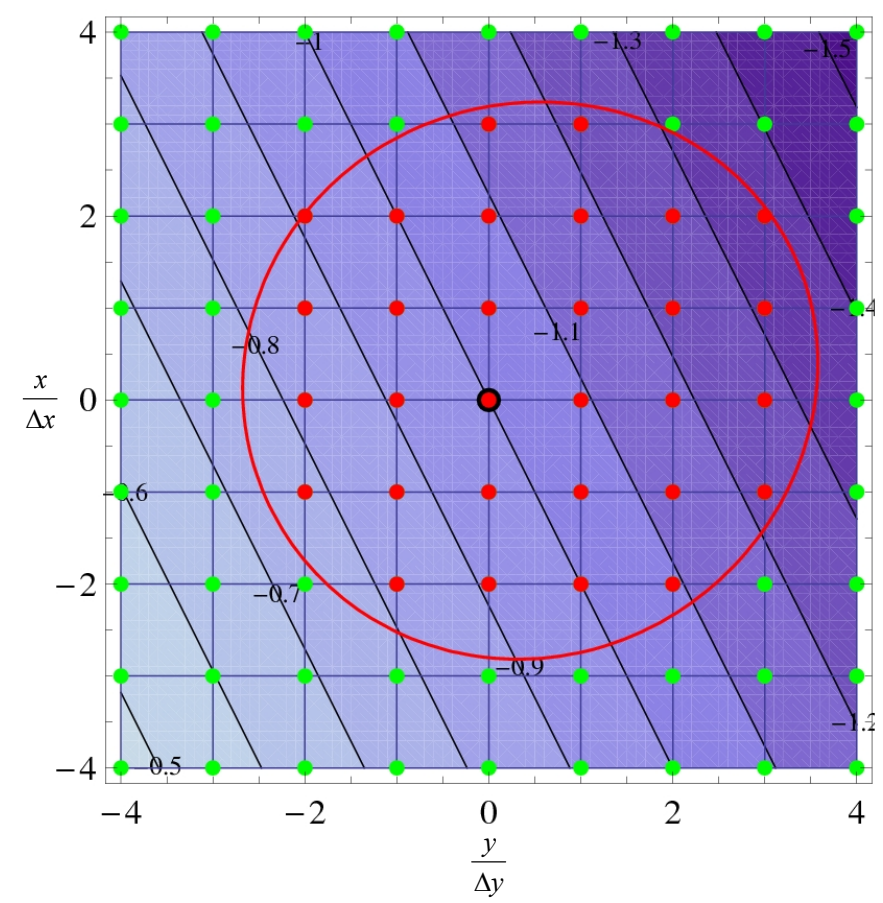

Figure 5. The contours and shading show the plane sloping depth as given by (20). The dots show nodes of a non-staggered grid and the red dots show the convolution stencil for $s_{0}=3$, which in turn corresponds to the limiting contour of the variable-depth impulse response function shown as the red oval.

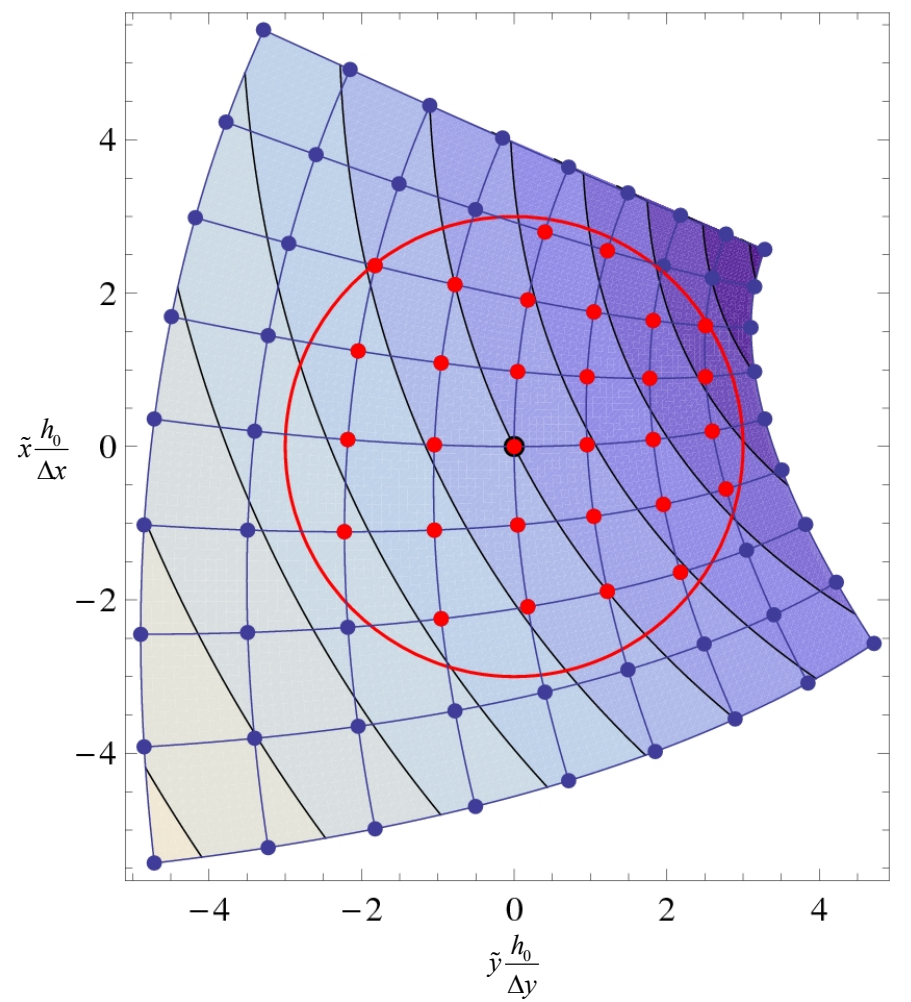

Figure 6. As Figure 5, but in the slightly distorted physical space in which the contours of the impulse response function have radial symmetry as shown by the circle of radius $s_{0}=3$. 
then the depth contours are shown in Figure 5 along with the nodes of a uniform (non-staggered) grid. The distorted grid is now computed according to the above and the nodal points are shown in Figure 6. Although all the depth information is now contained in $(\tilde{x}, \tilde{y})$, this figure also shows the distorted depth contours. In the distorted physical space the continuous impulse response function corresponding to the transfer function $(\tanh \kappa) / \kappa$ has circular symmetry as illustrated by the red circle in Figure 6 . Here we write $\kappa$ in place of $k h$ due to the associated physical-space distortion. The least squares fit is now made while including points within a certain radius as shown in red. In the original physical space these points lie within an oval, with points as well as oval shown in red in Figure 5. As it shows, more points are needed in the deeper parts than in the shallower parts consistent with the fact that decay distance of the impulse response function scales with the water depth.

Making the exercise of the least squares fit according to the outline above, we arrive at the results shown in Figure 7, which need a little more explanation. While the linear dispersion relation for constant depth is given by

$$
\frac{c^{2}}{g h}=\frac{\tanh (k h)}{k h}
$$

where $c$ is the wave celerity, we define a similar quantity $\tilde{c}$ for the variable depth by

$$
\frac{\tilde{c}^{2}}{g h}=\frac{\tanh (\kappa)}{\kappa}
$$

The ordinate axis in Figure 7 is the fitted value of this quantity relative to the target. The abscissa is $\kappa \Delta x / h_{0}$ which is close to $k \Delta x$. The concept of a Nyquist wavenumber is blurred by the slightly nonequidistant nodal spacing, but the range of the abscissa is chosen as $0<\kappa \Delta x / h_{0}<\pi$. The fit was made using uniform weights up to $\left|\kappa \Delta x / h_{0}\right|=2 \pi / 3$ and zero weights above this value. The limit corresponds to roughly three points per wavelength. The green curves show results for various wave directions using points within the circle of radius $s_{0}=3$ in the $\left(\tilde{x} h_{0} / \Delta x, \tilde{y} h_{0} / \Delta x\right)$-plane as shown in Figure 6 . The maximum error for the green lines in Figure 7 is as about $1 \%$ within the target range, $\left|\kappa \Delta x / h_{0}\right|=2 \pi / 3$. This error is small compared with the usual rule of thumb for Pade[2,2] Boussinesq formulations where traditionally a $5 \%$ error on the squared celerity has been taken as the acceptable limit. Increasing the radius to $s_{0}=3 \sqrt{2}$ and thus roughly doubling the number of convolution coefficients, the error decreases by an order of magnitude as shown by the blue lines in Figure 7. This illustrates how the error can be controlled by adjusting the size of the convolution stencil.

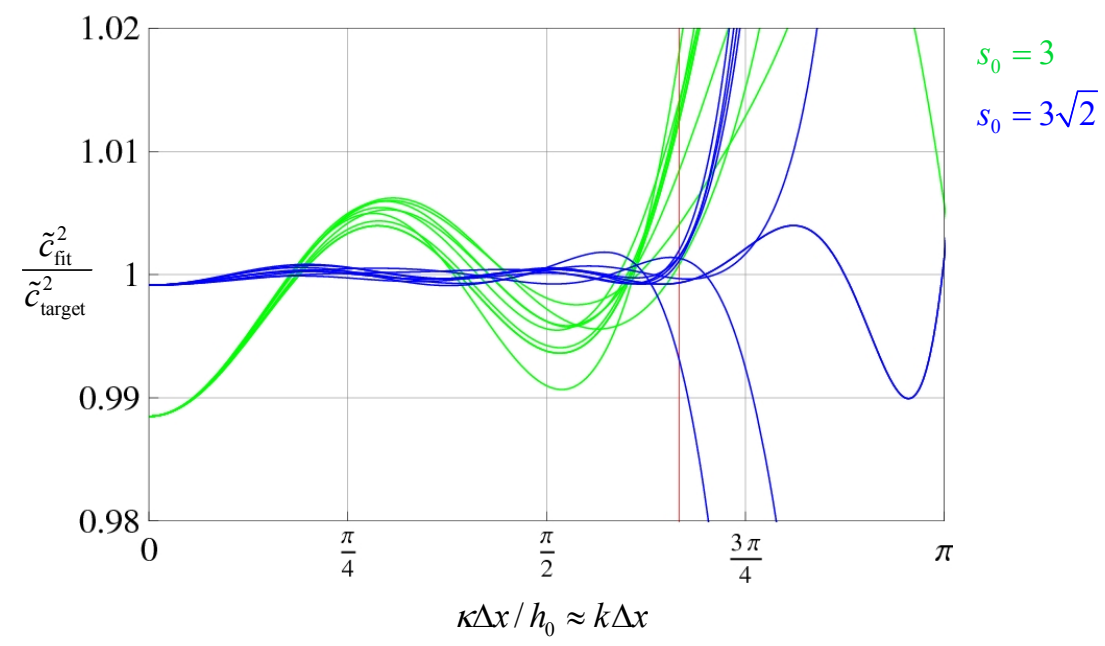

Figure 7. The error factor of the variable-depth fit for various wave directions using two different sizes of the convolution stencil. 
Note that the above is a brief account of an ongoing development and it does not contain a full description of how the convolution generalizes to $2 \mathrm{DH}$ on variable depth, neither in regard to the continuous nor the discrete formulation. Also, above example only shows results for the discrete convolution operator while the discrete dispersion relation for the full model (as shown above for the case of 1DH) has not been treated.

\section{SUMMARY AND CONCLUSION}

Two aspects of an ongoing development of a convolution type deterministic wave model have been considered, the first one associated with the linear part of the model in 1DH and the other related to the discrete convolution in 2DH for variable depth.

With regard to the 1DH model, the continuous as well as the discrete model has been outlined along with the associated discrete dispersion relation. The solution to the discrete dispersion relation has been shown to accurately predict the results of a numerical simulation. This suggests that numerical model parameters are determined on the basis of acceptable global errors rather than rules of thumb as often applied e.g. in Boussineq type modeling. It is recommended that this line of thinking is adopted in connection with other models as well. In the case of the convolution approach it was further mentioned how the CPU time can be minimized while balancing the effort in the various components of the model while adhering to given error limits.

As an introduction to the case of $2 \mathrm{DH}$, the discretization of the $1 \mathrm{DH}$ convolution by straightforward staggered-grid sampling of the impulse response function has been analyzed. The reason for the success of this approach in $1 \mathrm{DH}$ has been pinpointed and it has been shown that the particular advantage related to aliasing does not carry over to $2 \mathrm{DH}$. This calls for a new line of thinking in $2 \mathrm{DH}$ and a promising approach has been outlined. This approach focuses on the wave-number space transfer function rather than the physical-space impulse response function. Variable depth is accounted for by a slight distortion of physical space. This distortion has the purpose of rendering an associated wavenumber space in which the target transfer function retains the form that applies for the constantdepth situation. For constant depth, the distortion reduces to a simple depth scaling. The method involves a least squares procedure for the determination of the convolution coefficients needed in the $2 \mathrm{DH}$ discrete convolution and a theoretical example for a plane sloping bottom is given to support the feasibility of the approach.

\section{ACKNOWLEDGMENTS}

Support from the Danish Council for Independent Research | Technology and Production Sciences (FTP 09-065781) is greatly appreciated.

\section{REFERENCES}

Bingham, H.B., Agnon, Y., 2005. A Fourier-Boussinesq method for nonlinear water waves. Eur. J. Mech. B, Fluids 24, 255-274.

Matsuno, Y., 1993. Nonlinear evolution of surface gravity waves over an uneven bottom. Journal of Fluid Mechanics 249, 121-133.

Otta, A.K., Dingemans, M.W., Radder, A.C., 1996. A Hamiltonian model for nonlinear water waves and its applications. Proceedings of $25^{\text {th }}$ International Conference on Coastal Engineering, ASCE, 1156-1167.

Radder, A.C., 1992. An explicit Hamiltonian formulation of surface waves in water of finite depth, Journal of Fluid Mechanics, 237, 435-455.

Schäffer, H.A., 2005. A convolution method for 1-D nonlinear dispersive wave transformation over a mild-slope bottom. In: Edge, B.L., Santas, C.C. (Eds.), Waves '05: Proceedings of $5^{\text {th }}$ Int. Symp. on Ocean Wave Measurement and Analysis, Madrid, Spain, ASCE.

Schäffer, H.A., 2006. Progress on a convolution method for nonlinear, dispersive wave transformation over gently varying bathymetry. Proceedings of $30^{\text {th }}$ International Conference on Coastal Engineering, ASCE, 149-156.

Schäffer, H.A., 2008a. Toward a fast convolution-type wave model in non-rectangular domains. Proceedings of $31^{\text {st }}$ International Conference on Coastal Engineering, ASCE, 181-190.

Schäffer, H.A., 2008b. Comparison of Dirichlet-Neumann operator expansions for nonlinear surface gravity waves. Coastal Engineering, 55, 288-294.

Schäffer, H.A., 2009. A fast convolution approach to the transformation of surface gravity waves: Linear waves in 1DH, Coastal Engineering, 56, 517-533. 